\title{
Synthesis of ZnO Nanoparticles at Low Temperature by Hydrothermal Treatment Using the Sol-Gel Method
}

\author{
B. Murguía-Martínez ${ }^{1}$, S.E. Borjas-García ${ }^{2}$, A. Medina ${ }^{*}$, L. Béjar ${ }^{3}$, P. Martínez-Torres ${ }^{2}$ \\ 1. Instituto de Investigación en Metalurgia y Materiales, UMSNH, Morelia, Michoacán, México. \\ 2. Instituto de Física y Matemáticas, UMSNH, Morelia, Michoacán, México. \\ 3. Facultad de Ingeniería Mecánica, UMSNH, Morelia, Michoacán, México. \\ *Corresponding author: ariostomedina@gmail.com
}

Research on nano-crystalline materials has been increased enormously in recent years. Because the several areas of application for this new type of materials in nanometer scale. These nano-scale properties depend on the size and appear when the size of the particles is approximately Bohr's diameter for the exciton [1]. Zinc oxide $(\mathrm{ZnO})$ is an n-type semiconductor that shows a hexagonal wurtzite crystal structure, with a P63mc space group and parameters of $\mathrm{a}=\mathrm{b}=0.3250 \mathrm{~nm}$ and $\mathrm{c}=0.5207 \mathrm{~nm}$ with quantum confinement effects. For the synthesis of zinc oxide nanoparticles, the sol-gel method showed several advantages over other techniques, due to the good stability of the solution, low temperature of hydrothermal treatment, besides not adding any type of surfactant or doping agent [2].

The nanoparticles of zinc oxide of this work were prepared by using zinc nitrate hexahydrate (Aldrich), citric acid (Aldrich) and sodium hydroxide (J. T. Baker). In the first step, three solutions were prepared. The first one was prepared by dissolving $0.04 \mathrm{~mol}$ of citric acid in $0.6 \mathrm{~mol}$ of distilled water. The second one was prepared by dissolving $0.02 \mathrm{~mol}$ of zinc precursor in $0.6 \mathrm{~mol}$ of distilled water and, the third one by dissolving $0.04 \mathrm{~mol}$ of sodium hydroxide in $1.2 \mathrm{~mol}$ of distilled water. For the second step, the zincsolution was added slowly (drop by drop) to citric acid solution. Then, Na-solution was added slowly (drop by drop) to zinc-citric acid solution and vigorous stirred for $30 \mathrm{~min}$, too. The gel obtained was aged in a closed glass flask at $100{ }^{\circ} \mathrm{C}$ for 1 day. After, the hydrothermal treatment, the sample was washed with distilled water and centrifuged at $12000 \mathrm{rpm}$ for 10 minutes. Then, the material was dried at $80{ }^{\circ} \mathrm{C}$ for 1 day. Finally, it was necessary to calcine the sample at $560^{\circ} \mathrm{C}$ for $1 \mathrm{~h}$ to eliminate the residual organic.

Figure 1 shows the results of X-rays analysis, peaks of high intensity corresponding to the zinc oxide (after calcination of sample), there was not observed any other phase present, which indicates a high degree of purity. In addition, the $\mathrm{ZnO}$ crystals have a hexagonal crystal lattice wurtzite type, this structure is the most stable for zinc oxide, no preferential orientation is observed in the diffraction pattern [3].

Figure 2 shows the shape of the nanoparticles analyzed by SEM, the hydrolysis-condensation is not controlled. There is no preferential growth along the c axis, for that, the particles have spherical shape. Citric acid limits the growth of the particles within the nucleation in the solution. As shown in Figures $2 \mathrm{a}$ and $2 \mathrm{~b}$, the high agglomeration of the nanoparticles was since during the formation of $\mathrm{ZnO}$ each $\mathrm{Zn}^{2+}$ ion would undergo a chelation with $\mathrm{C}_{2} \mathrm{O}_{2}^{-4}$ ligand to form a stable complex, thus decreasing the aggregation of particles in the nucleation process [4]. 
References:

[1] M. Bangal et al., Springer vol. 160 (2005), pp 81-94.

[2] R. M. Alwan et al., Sapub vol. 5 (2015), pp 1-6.

[3] M. M. Ba-Abbad et al., Elsevier vol. 550 (2013), pp 63-70.

[4] R. Wahab et al., Materials Transactions vol. 50 (2009), pp2092-2097.

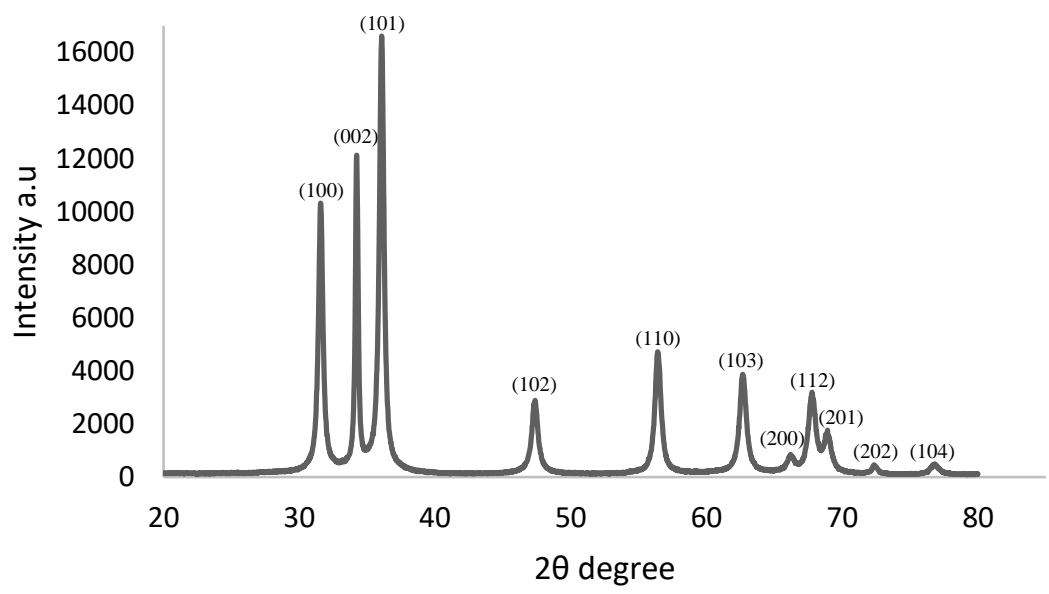

Figure 1. X-ray analysis of nanoparticles of zinc oxide.
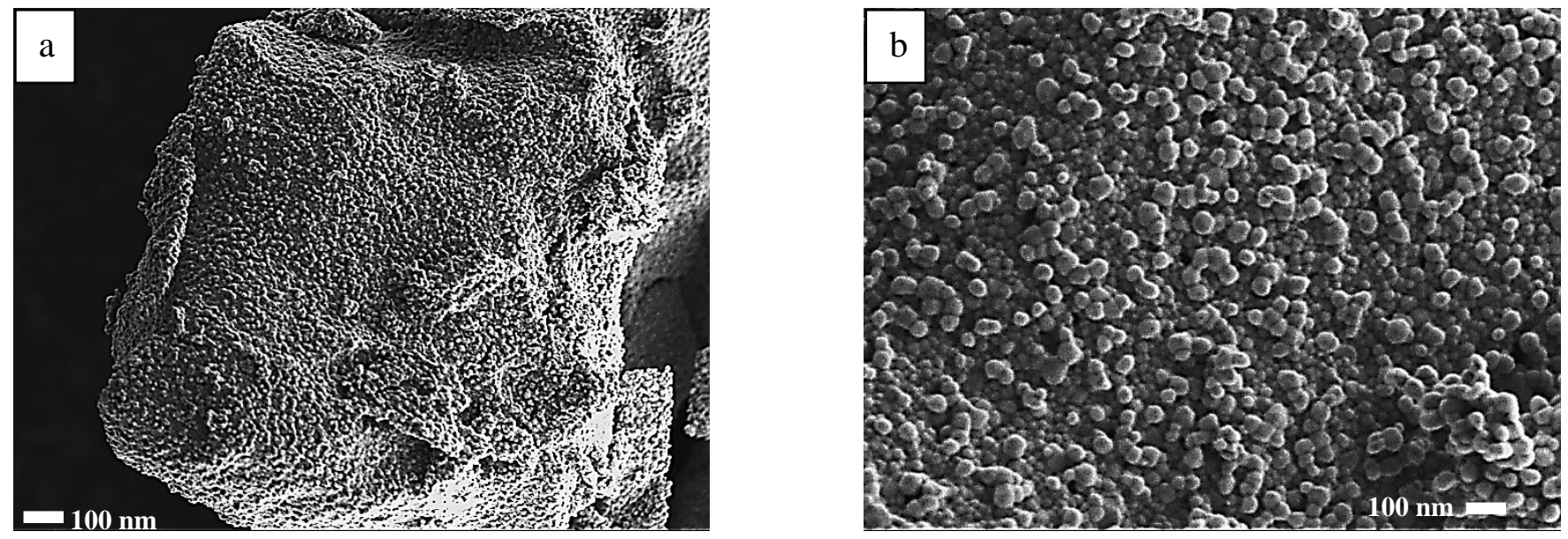

Figure 2. SEM images of nanoparticles of zinc oxide synthesized at $100{ }^{\circ} \mathrm{C}$, a) $10000 \mathrm{x}$, and b) $40000 \mathrm{x}$, magnification. 